\title{
Fitossociologia de um brejo de altitude no semiárido brasileiro: variação das espécies dominantes ao longo do gradiente altitudinal
}

\author{
Plant communities in a "brejo de altitude" of the brazilian semiarid: change of \\ dominants species along the elevation gradient
}

\author{
Thallyta Guimarães Araujo ${ }^{I}$, Augusto Barbosa de Queiroz ${ }^{\text {II }}$, Sérgio de Faria Lopes ${ }^{\text {III }}$
}

\section{Resumo}

Neste estudo, objetivou-se caracterizar a composição e estrutura da comunidade arbórea da Serra dos Cavalos, em Pernambuco, buscando inferir sobre a influência do gradiente altitudinal na variação das espécies de maior Valor de Importância (VI). Para isto, foi realizado um estudo fitossociológico em que foram mensurados todos os indivíduos vivos com circunferência a altura do peito (CAP) $\geq 15 \mathrm{~cm}$. Foram avaliados os descritores fitossociológicos clássicos em três diferentes níveis altitudinais (base, nível intermediário e topo). Foram registrados 1.500 indivíduos ao longo de toda a serra, distribuídos em 56 espécies e 29 famílias. O primeiro nível altitudinal (N1) apresentou 44 espécies, no nível intermediário (N2) foram encontradas 37 espécies e, no terceiro nível (N3), 41 espécies. A diferença na composição das espécies ao longo da altitude foi pequena. Os valores de densidade diferiram entre os níveis, com maior densidade no N2, possivelmente devido a frequentes perturbações antrópicas. Entre as dez espécies de maior valor de importância (VI) para cada nível altitudinal, Byrsonima sericea DC., Eriotheca crenulaticalyx A. Robyns, Tapirira guianensis Aubl. e Ocotea glomerata (Ness) Mez são espécies comuns para as três altitudes, porém, alternam a posição do VI ao longo da serra. A variação dos descritores fitossociológicos entre os níveis pode ser consequência da variação da importância destas espécies, resultado das modificações ambientais características de cada nível de elevação. O sucesso em explorar os recursos por parte dessas espécies em todos os níveis contribuiu para a alta similaridade florística em todo o gradiente, além de definir a estrutura dessa floresta de brejo.

Palavras-chave: Floresta montana; Serra dos Cavalos; Nível altitudinal; Comunidade arbórea

\begin{abstract}
This study aims to characterize the composition and structure of Serra dos Cavalos tree community, in Pernambuco, trying to infer the influence of altitudinal gradient on the variation of the dominant species. For this, it carried out a phytosociological study, which were measured living individuals with circumference at breast height $(\mathrm{CBH})>15 \mathrm{~cm}$. Was evaluated the classical phytosociological parameters in three different altitudinal levels. Were recorded 1500 individuals throughout the mountain range, distributed in 56 species 29 families and. The first altitudinal level presented 44 species; in mid-level 37 species were found and in level 3, 41 species. The difference in species composition along the altitude was small. The density values differed among levels, with greater density in N2, possibly due to frequent human disturbance. Level 3 was sampled at the highest value of basal area. Among the ten species of greatest importance value (VI) for each level species in each altitudinal level, Byrsonima sericea DC., Eriotheca crenulaticalyx A. Robyns, Tapirira guianensis Aubl. and Ocotea glomerata (Ness) Mez are common to the three altitudes, but be alternation of dominance along the mountain range. The variation of phytosociological parameters among the levels, which can be a result of dominance of these species, in addition to a change of environmental characteristics at each level. The success in exploiting the resources of these species for all levels contributed to the high floristic similarity across the gradient, and defines the structure of this swamp forest.
\end{abstract}

Keywords: Montane forest; Cavalos mountain range; Altitudinal level; Tree community

Bióloga, MSc., Programa de Pós-Graduação em Ecologia e Conservação, Universidade Estadual da Paraíba, Rua Baraúnas, 351, Bairro Universitário, CEP 58430 335, Campina Grande (PB), Brasil. tga13011988@gmail.com (ORCID: 0000-0001-9820-7226)

Doutorando em Ecologia do Programa de Pós-Graduação em Ecologia e Recursos Naturais da Universidade Federal do Ceará, Brasil. abqueiiroz@ gmail.com (ORCID: 0000-0001-6084-3956)

III Biólogo, Dr., Professor do Departamento de Biologia, Universidade Estadual da Paraíba, Rua Baraúnas, 351, Bairro Universitário, CEP 58430 335, Campina Grande (PB), Brasil. defarialopes@gmail.com (ORCID: 0000-0001-6472-6765) 


\section{Introdução}

As florestas montanas do nordeste do Brasil ocorrem em topos e encostas com altitudes que variam entre 600 a 1.100 m a.n.m. (VASCONCELOS SOBRINHO, 1970). Estas florestas serranas, também chamados de brejos de altitude, são enclaves no bioma Mata Atlântica em áreas de maiores altitude e umidade em relação à matriz de vegetação xerófila (Caatinga) (CAVALCANTI; TABARELLI, 2004). Os brejos são, em quase toda sua totalidade, disjunções de floresta estacional semidecidual montana, um dos tipos vegetacionais que compõem o bioma Mata Atlântica (IBGE, 2012). A presença dessa vegetação florestal no domínio do semiárido ocorre principalmente devido ao fator topográfico que permite um incremento na média da pluviosidade, em relação às áreas que as circundam (TAVARES et al., 2000).

De acordo com Gentry (1998), mudanças florísticas e estruturais em florestas neotropicais ocorrem ao longo de gradientes de altitudes, das terras baixas até montanas. Em gradientes altitudinais, como os brejos de altitude do semiárido brasileiro, a composição e estrutura da vegetação pode estar relacionado às variações de habitat em escala local (IVANAUSKAS; MONTEIRO; RODRIGUES, 2000; MORELLATO, 2000), determinado pelo microclima (PROCTOR et al., 1988; MONTEIRO; FISCH, 2005), solo, topografia (OLIVEIRA FILHO et al., 1999; CARVALHO et al., 2005) e nível de declividade (OLIVEIRA; MORI, 1999), além de interações interespecíficas (WHITTAKER; WILLIS; FIELD, 2001).

A heterogeneidade ambiental em gradientes de altitude favorece uma variação das espécies vegetais dominantes (por densidade ou biomassa) ao longo do gradiente (WEBB; PEART, 2000; ZHAO et al., 2005). Fatores físicos e bióticos atuam sobre o ambiente, modificando as características e regulando o mosaico vegetacional (IVANAUSKA; MONTEIRO; RODRIGUES, 2000; MORELLATO, 2000). Em um estudo com 20 fragmentos de florestas secundárias de Mata Atlântica no sudeste do Brasil com pouca variação na altitude $(150 \mathrm{~m})$, evidenciou maior riqueza e cobertura florestal em altitudes superiores, além de um menor grau de perturbação (SILVA et al., 2008). Outros estudos, apontam variações nos parâmetros fitossociológicos ao longo de gradientes altitudinais, como exemplo, a variação na abundância relativa (BOTREL et al., 2002), ou mesmo relacionado ao valor de importância (VI) das espécies de acordo com o nível de altitude (GOMES; BERNACCI; JOLY, 2011).

O brejo de altitude no município de Caruaru, Pernambuco, conhecido como Parque Ecológico João Vasconcelos Sobrinho foi indicado como uma reserva de prioridade máxima para a conservação segundo o Workshop sobre Áreas Prioritárias para a Conservação da Mata Atlântica do Nordeste (FUNDAÇÃO SOS MATA ATLÂNTICA, 1993). Dessa forma, estudos que buscam entender as variações na composição e estrutura fitossociológica deste brejo de altitude nos diferentes níveis de altitude são relevantes para subsidiar ações conservacionistas para este tipo vegetacional endêmico do nordeste brasileiro.

Neste contexto, o estudo objetivou-se caracterizar a composição e estrutura da vegetação lenhosa de um brejo de altitude pernambucano, analisando a variação das comunidades arbóreas em três níveis de altitude. Com o intuito de responder as seguintes questões: (i) existem diferenças na composição e estrutura fitossociológica das comunidades entre os níveis de altitude analisados? (ii) as espécies mais importantes da serra (maiores VI) são as mesmas nos diferentes níveis de altitude? As respostas a estas questões poderão subsidiar propostas voltadas a eleger áreas prioritárias para a conservação da Mata Atlântica no Estado.

\section{Material e métodos}

A área estudada está localizada no Parque Ecológico João Vasconcelos Sobrinho (08²2’09” S e 3605’00” W), situado no município de Caruaru - PE. (Figura 1). A serra analisada apresenta altitude máxima de $970 \mathrm{~m}$. O clima da área é tropical chuvoso com verão seco (As'), segundo a classificação atualizada de Köppen-Geiger (PEEL; FINLAYSON; MCMAHON, 2007) 
com temperatura média de $24^{\circ} \mathrm{C}$ (AGÊNCIA ESTADUAL DE MEIO AMBIENTE E RECURSOS HÍDRICOS, 1994). A precipitação média anual oscila entre 650 e $800 \mathrm{~mm}$, com um maior índice pluviométrico entre os meses de abril e junho, com estação seca variando de cinco a sete meses (AGÊNCIA ESTADUAL DE MEIO AMBIENTE E RECURSOS HÍDRICOS, 1994).

Figura 1 - Mapa de localização do Brejo dos Cavalos, Pernambuco, Brasil.

Figure 1 - Location map of the Brejo dos Cavalos, Pernambuco state, Brazil.

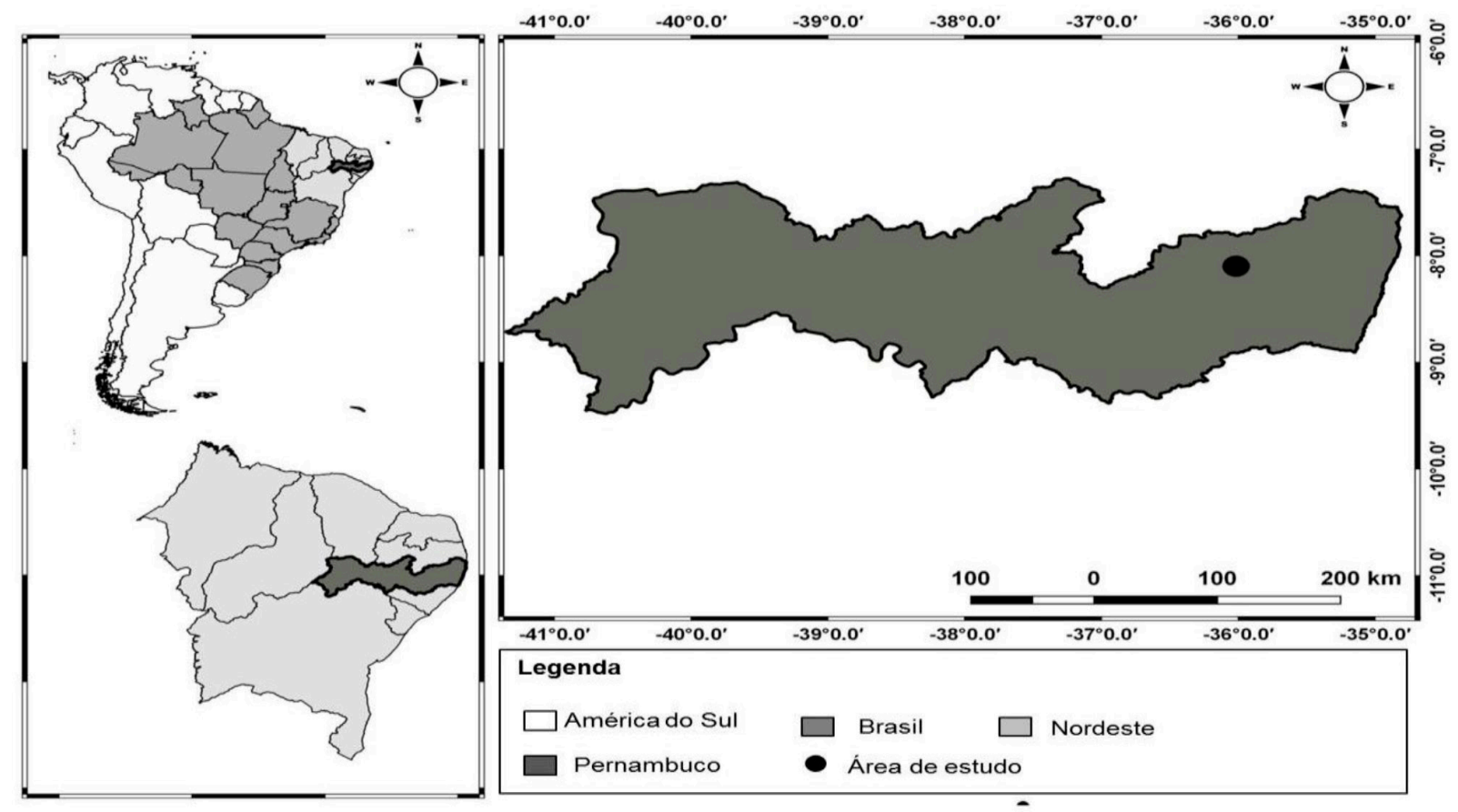

Fonte: Maiara Bezerra Ramos, Laboratório de Ecologia \& Conservação de Florestas Secas - EcoTropics, UEPB. 2018 Source: Maiara Bezerra Ramos, Laboratory of Ecology \& Conservation of Dry Forest - EcoTropics, UEPB. 2018.

A maior parte do parque é coberto por floresta estacional semidecidual montana, com um dossel em torno de $20 \mathrm{~m}$ de altura (TAVARES et al., 2000). Segundo informações de moradores locais, a área era ocupada por plantios de café até meados da década de 40 do século XX. Os solos são profundos, predominando o podzólico vermelho-amarelo distrófico latossólico no topo das serras e podzólico vermelho-amarelo distrófico nas encostas; planossolo solódico, circundando a área serrana e gley húmico e pouco húmico nos vales das serras (AGÊNCIA ESTADUAL DE MEIO AMBIENTE E RECURSOS HÍDRICOS, 1994). Quanto à geologia, o parque localiza-se em terrenos de origem pré-cambriana pertencentes ao complexo cristalino da Borborema, formado por granodioritos com gradação para granitos e tenolitos de coloração e granulometria variada. No que se refere ao aspecto geomorfológico, a área está representada por relevos residuais sobre superfícies pediplanadas, as chamadas serras, com relevo que varia de ondulado a forte ondulado (TAVARES et al., 2000)

Com auxílio de aparelho GPS (Global System Position) foram demarcados três níveis de altitude ao longo de toda área serrana, sendo Nível 1 (N1) com elevação de 800 a $850 \mathrm{~m}$ a.n.m. (base da serra), Nível 2 (N2) com elevação variando entre 851 até $900 \mathrm{~m}$ (nível intermediário), e Nível 3 (N3) de 901 a 950 m (topo da serra). A divisão da serra em três níveis de altitude foi baseada em trabalhos realizados anteriormente por Oliveira et al. (2009), Silva et al. (2014) e Lopes, Ramos e Almeida (2017) que sugeriram existir diferenças florísticas e estruturais entre a base, níveis de altitude intermediários e topo de serra. 
Para a amostragem da vegetação foi utilizado o método de quadrante centrados (COTTAM; CURTIS, 1956). Foram mensurados quatro indivíduos arbóreos vivos e com circunferência a altura do peito $(\mathrm{CAP}) \geq 15 \mathrm{~cm}$, bem como a altura e a distância entre o ponto quadrante à árvore mais próxima, presente dentro de cada quadrante. As amostras foram constituídas em 125 pontos aleatórios e correspondente a cada nível altitudinal (N1, N2 e N3), totalizando 375 pontos amostrais com uma distância de 10 metros entre os pontos.

Para a caracterização da vegetação arbórea, foram calculados para cada espécie, os valores de densidade, dominância e frequência relativas, valor de importância (VI) e área basal total. As análises de similaridade florística (presença ou ausência) e de estrutura (abundância) foram realizadas pelos cálculos dos índices de Sorensen, Jaccard (dados binários) e Bray Curtis (dados de abundância), utilizando o software Fitopac Shell, versão 2.1. (SHEPHERD, 2010). Para a avaliação da diversidade foram calculados os índices de diversidade de Shannon (H') e de equabilidade de Pielou (J'). Os índices de diversidade em cada nível altitudinal foi comparado pelo teste-t de Hutchinson, utilizando o Programa estatístico PAST (HAMMER; HARPER; RYAN, 2001). As identificações foram realizadas por meio de pareceres de especialistas e literatura especializada. As espécies foram classificadas nas famílias de acordo com o sistema APG III (ANGIOSPERM PHYLOGENY GROUP, 2009).

\section{Resultados e discussão}

\section{Florística}

Foram amostrados 1.500 indivíduos, pertencentes a 56 espécies e 31 famílias, em 1,5 ha ao longo da serra. O primeiro nível altitudinal (N1) apresentou 44 espécies e 22 famílias; no N2 foram amostradas 37 espécies e 24 famílias e no N3 foram encontradas 41 espécies e 24 famílias (Tabela 1). As famílias mais representativas foram Fabaceae (11 espécies), Anacardiaceae (quatro espécies), Lauraceae e Rubiaceae com três espécies e Clusiaceae, Combretaceae, Malpighiaceae, Meliaceae, Moraceae e Myrtaceae com duas espécies cada. Estas famílias também são aquelas frequentemente encontradas com maior riqueza de espécies em outros estudos realizados em brejos de altitude no Brasil (ANDRADE et al., 2006; OLIVEIRA; ANDRADE; FELIX, 2006; NASCIMENTO; RODAL, 2008). Estas dez famílias são responsáveis por 53,6\% de todas as espécies levantadas no presente estudo, observaram-se também seis espécies exóticas, o que pode estar associado ao grau de conservação da área. Entre os táxons representantes da área amostrada Albizia polycephala, Buchenavia capitata, Casearia sylvestris, Myrcia sylvicata e Tapirira guianensis, são espécies frequentemente citadas em levantamentos florísticos realizados nas florestas serranas nordestinas (Tabela 1).

\section{Tabela 1 - Lista de famílias e espécies amostradas e respectivas abundâncias por nível altitudinal no Brejo dos Cavalos, Pernambuco, Brasil}

Table 1 - List of families and species and their abundances by altitudinal level in the Brejo dos Cavalos, Pernambuco state, Brazil

\begin{tabular}{|c|c|c|c|c|}
\hline Família/Espécie & Nome popular & Nível 1 & Nível 2 & Nível 3 \\
\hline \multicolumn{5}{|l|}{ ANACARDIACEAE } \\
\hline Anacardium occidentale L. & cajueiro & 4 & 0 & 0 \\
\hline Tapirira guianensis Aubl. & pau-pombo & 71 & 101 & 101 \\
\hline
\end{tabular}


Tabela 1 - Continuação...

Table 1 - Continuation...

\begin{tabular}{|c|c|c|c|c|}
\hline Família/Espécie & Nome popular & Nível 1 & Nível 2 & Nível 3 \\
\hline Thyrsodium schomburgkianum Benth. & cabotã & 60 & 4 & 15 \\
\hline Schinopsis brasiliensis Engl. & maria-preta & 1 & 0 & 2 \\
\hline \multicolumn{5}{|l|}{ APOCYNACEAE } \\
\hline Aspidosperma sp. & pereiro & 0 & 1 & 0 \\
\hline \multicolumn{5}{|l|}{ ARALIACEAE } \\
\hline $\begin{array}{l}\text { Schefflera morototoni (Aubl.) Maguire- } \\
\text { Steyerm. \& Frodin }\end{array}$ & sabaquim & 0 & 3 & 4 \\
\hline \multicolumn{5}{|l|}{ ARECACEAE } \\
\hline Attalea oleifera Barb. Rodr. & palmeira & 1 & 0 & 2 \\
\hline \multicolumn{5}{|l|}{ BORAGINACEAE } \\
\hline Cordia sellowiana Cham. & gaiúba & 2 & 2 & 12 \\
\hline \multicolumn{5}{|l|}{ CLUSIACEAE } \\
\hline Clusia dardanoi G. Mariz \& Maguire & $\begin{array}{l}\text { orelha-de- } \\
\text { onça }\end{array}$ & 3 & 29 & 18 \\
\hline Vismia guianensis (Aubl.) Choisy & lacre & 8 & 0 & 5 \\
\hline \multicolumn{5}{|l|}{ COMBRETACEAE } \\
\hline Buchenavia capitata (Vahl) Eichl. & esparrada & 0 & 1 & 0 \\
\hline Combretum sp. & rama-branca & 1 & 6 & 7 \\
\hline \multicolumn{5}{|l|}{ CHRYSOBALANACEAE } \\
\hline $\begin{array}{l}\text { Licania octandra (Hoffmanns. ex Roem. \& } \\
\text { Schult.) Kuntze }\end{array}$ & goití & 0 & 0 & 1 \\
\hline \multicolumn{5}{|l|}{ ERYTHROXYLACEAE } \\
\hline Erythroxylum mucronatum Benth. & cafezinho & 1 & 6 & 2 \\
\hline \multicolumn{5}{|l|}{ EUPHORBIACEAE } \\
\hline Mabea occidentalis Benth. & $\begin{array}{l}\text { canudo- } \\
\text { caximbo }\end{array}$ & 0 & 0 & 6 \\
\hline \multicolumn{5}{|l|}{ FABACEAE } \\
\hline Albizia polycephala (Benth.) Killip ex Record & jaguarana & 1 & 11 & 6 \\
\hline Andira fraxinifolia Benth. & piaca & 1 & 0 & 0 \\
\hline Andira nitida Mart. ex Benth. & angelin & 2 & 1 & 2 \\
\hline Copaifera langsdorffii Desv. & pau-de-óleo & 0 & 0 & 1 \\
\hline Inga sp. & ingá & 32 & 1 & 3 \\
\hline Machaerium hirtum (Vell.) Stellfeld & chifre-de-bode & 2 & 7 & 3 \\
\hline Ormosia fastigiata Tul. & sucupira & 13 & 28 & 8 \\
\hline
\end{tabular}


Tabela 1 - Continuação...

Table 1 - Continuation...

\begin{tabular}{|c|c|c|c|c|}
\hline Família/Espécie & Nome popular & Nível 1 & Nível 2 & Nível 3 \\
\hline Piptadenia stipulacea (Benth.) Ducke & jurema-preta & 1 & 0 & 0 \\
\hline Pterocarpus violaceus Vogel & pau-sangue & 0 & 0 & 2 \\
\hline $\begin{array}{l}\text { Stryphnodendron pulcherrimum (Willd.) } \\
\text { Hochr. }\end{array}$ & favinha & 8 & 9 & 8 \\
\hline Swartzia pickelli Killip. ex Ducke & jacarandá & 5 & 11 & 3 \\
\hline \multicolumn{5}{|l|}{ LAURACEAE } \\
\hline Nectandra cuspidata Nees \& Mart. & louro-canela & 16 & 3 & 17 \\
\hline Ocotea gardneri (Meins.) Mez & louro-babão & 0 & 0 & 6 \\
\hline Ocotea glomerata (Ness) Mez & louro & 32 & 37 & 45 \\
\hline \multicolumn{5}{|l|}{ MALPIGHIACEAE } \\
\hline Byrsonima crispa A. Juss. & muricí-branco & 1 & 0 & 0 \\
\hline Byrsonima sericea DC. & muricí & 46 & 63 & 17 \\
\hline \multicolumn{5}{|l|}{ MALVACEAE } \\
\hline Eriotheca crenulaticalyx A. Robyns & munguba- & 8 & 40 & 23 \\
\hline Melochia sp. & vermelhinho & 2 & 0 & 0 \\
\hline \multicolumn{5}{|l|}{ MELASTOMATACEAE } \\
\hline Miconia munutiflora (Bonpl.) DC. & carrasco & 0 & 7 & 15 \\
\hline \multicolumn{5}{|l|}{ MELIACEAE } \\
\hline Cedrela fissilis Vell. & cedro & 4 & 0 & 2 \\
\hline Guarea macrophylla Vahl & gitó & 13 & 2 & 7 \\
\hline \multicolumn{5}{|l|}{ MORACEAE } \\
\hline Artocarpus heterophyllus Lam. & jaca & 40 & 21 & 0 \\
\hline Ficus maxima Mill. & gameleira & 0 & 0 & 3 \\
\hline \multicolumn{5}{|l|}{ PRIMULACEAE } \\
\hline Myrsine guianensis (Aubl.) Kuntze & carne-de-vaca & 1 & 2 & 0 \\
\hline \multicolumn{5}{|l|}{ MYRTACEAE } \\
\hline Eugenia punicifolia (Kunth) DC. & murta & 18 & 2 & 35 \\
\hline Myrcia sylvatica (G. Mey.) DC. & prupuna & 9 & 29 & 9 \\
\hline \multicolumn{5}{|l|}{ NYCTAGINACEAE } \\
\hline Guapira laxiflora (Choisy) Lundell & piranha & 15 & 19 & 51 \\
\hline \multicolumn{5}{|l|}{ PODOCARPACEAE } \\
\hline Podocarpus sellowii Klotzsch ex Endl. & $\begin{array}{l}\text { pinheirinho- } \\
\text { bravo }\end{array}$ & 0 & 1 & 10 \\
\hline
\end{tabular}


Tabela 1 - Conclusão...

Table 1 - Conclusion...

\begin{tabular}{|c|c|c|c|c|}
\hline Família/Espécie & Nome popular & Nível 1 & Nível 2 & Nível 3 \\
\hline \multicolumn{5}{|l|}{ RUBIACEAE } \\
\hline Coffea arabica L. & café & 2 & 2 & 3 \\
\hline Genipa americana L. & jenipapo & 1 & 3 & 2 \\
\hline Palicourea crocea (Sw.) Roem. \& Schult. & erva-de-rato & 2 & 1 & 3 \\
\hline \multicolumn{5}{|l|}{ RUTACEAE } \\
\hline Zanthoxylum rhoifolium Lam. & limãozinho & 3 & 0 & 6 \\
\hline \multicolumn{5}{|l|}{ SALICACEAE } \\
\hline Casearia sylvestris Sw. & caiubim & 31 & 30 & 12 \\
\hline \multicolumn{5}{|l|}{ SAPINDACEAE } \\
\hline Talisia esculenta (Cambess.) Radlk. & pitomba & 1 & 0 & 0 \\
\hline \multicolumn{5}{|l|}{ SAPOTACEAE } \\
\hline Micropholis sp. & bom-nome & 0 & 5 & 21 \\
\hline \multicolumn{5}{|l|}{ SIMAROUBACEAE } \\
\hline Simarouba amara Aubl. & praíba & 4 & 6 & 2 \\
\hline \multicolumn{5}{|l|}{ URTICACEAE } \\
\hline Cecropia pachystachya Trécul & embaúba & 22 & 4 & 0 \\
\hline \multicolumn{5}{|l|}{ VERBENACEAE } \\
\hline Cytharexylum sp. & salgueiro & 3 & 0 & 0 \\
\hline \multicolumn{5}{|l|}{ VOCHYSIACEAE } \\
\hline Vochysia sp. & cinzeiro & 4 & 1 & 0 \\
\hline \multicolumn{5}{|l|}{ INDETERMINADAS } \\
\hline Morfospécie 1 & & 4 & 1 & 0 \\
\hline Morfospécie 2 & & 1 & 0 & 0 \\
\hline
\end{tabular}

Fonte: Thallyta Guimarães de Araujo, et al. 2018

A maior representatividade de Fabaceae na floresta estudada já era esperado, visto ser uma das famílias botânicas mais diversas em todas as províncias fitogeográficas do Brasil (CESTARO; SOARES, 2004), também comumente encontrada em áreas de floresta estacional semidecidual com alta densidade e riqueza (LOPES et al., 2012).

Avaliar as comunidades vegetais em um e/ou entre ambientes é uma ferramenta importante em estudos florísticos que visam ressaltar a diversidade florística. A análise da similaridade florística da vegetação associada pelos índices de Sorensen $(0,75-0,82)$ e Jaccard $(0,60-0,70)$ indicou uma alta similaridade entre os níveis altitudinais (Tabela 2). Esta similaridade também foi confirmada pelo valor encontrado com base no índice de Bray-Curtis $(0,17-0,24)$, indicando pequena dissimilaridade entre os níveis de altitude (Tabela 2). Do total de espécies, 44,64\% estavam distribuídas nos três níveis. Por outro lado, oito espécies foram exclusivas do N1, duas espécies do N2 e seis espécies do N3 (Figura 2). 
Tabela 2 - Análise de similaridade e dissimilaridade entre os níveis altitudinais, no Brejo dos Cavalos, Pernambuco, Brasil

Table 2 - Analysis of similarity and dissimilarity among altitudinal levels in the Brejo dos

Cavalos, Pernambuco state, Brazil

\begin{tabular}{lccc}
\hline Níveis & Sorensen & Jaccard & Bray Curtis \\
\hline $1 \times 2$ & 0,75 & 0,60 & 0,24 \\
$1 \times 3$ & 0,76 & 0,62 & 0,23 \\
$2 \times 3$ & 0,82 & 0,70 & 0,17 \\
\hline
\end{tabular}

Fonte: Thallyta Guimarães de Araujo, et al. 2018

A similaridade florística entre os três níveis calculadas pelos índices de Sorensen e Jaccard (Tabela 2), bem como pelo Diagrama de Veen (Figura 2), demostram uma flora comum entre os níveis, refletindo uma homogeneidade florística. Um estudo realizado também no estado de Pernambuco, em uma área de Caatinga, apresentou um alto índice de similaridade de Sorensen, mostrando que, apesar das diferenças de altitude das áreas analisadas, os ambientes são muito semelhantes (FARIAS et al., 2016). Esta alta similaridade encontrada entre os níveis pode estar relacionada às características ambientais intrínsecas da área, como os fatores edáficos (BRAGA; LIMA; MARTINS, 2015), luminosidade, temperatura, as quais possibilitam o estabelecimento das mesmas espécies (KELLMAN, 1980).

Figura 2 - Diagrama de Venn mostrando o número de espécies exclusivas e comuns a todos os níveis altitudinais analisados na Serra dos Cavalos, Pernambuco, Brasil

Figure 2 - Venn diagram showing the number of unique and common species to all altitudinal levels analyzed in Brejo dos Cavalos, Pernambuco state, Brazil

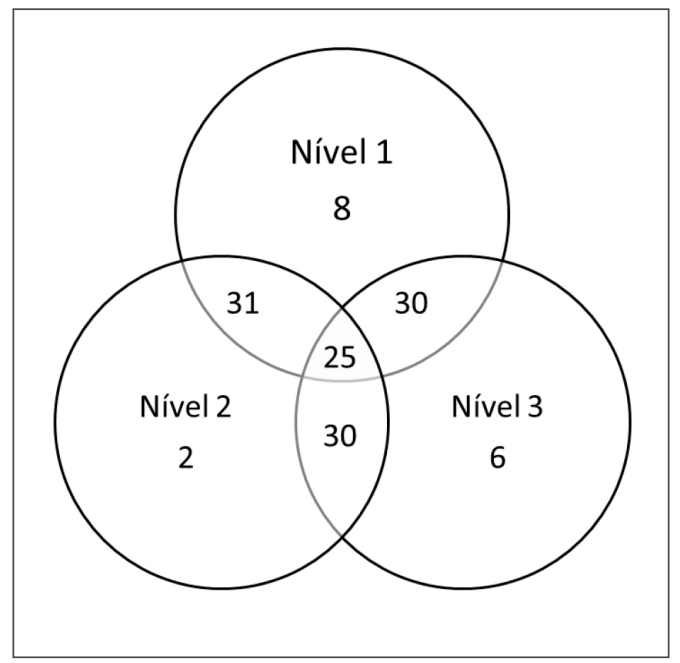

Fonte: Thallyta Guimarães de Araujo, et al. 2018

A distribuição das espécies na área estudada mostrou que a pequena variação na altitude resultou em uma elevada similaridade florística ao longo da serra, sendo necessárias diferenciações contrastantes nas condições do meio ambiente e um distanciamento maior entre os pontos amostrais, para que novas espécies possam ser amostradas. Embora a distância geográfica sozinha não responde totalmente pela substituição de espécies, pois diferenças 
ambientais e limites de dispersão das espécies podem ser responsáveis pela maior variação da composição florística entre áreas (TUOMISTO; RUOLOLAINEN; YLI-HALLA, 2003; DUQUE et al., 2009).

\section{Estrutura da vegetação}

Das 44 espécies amostradas no N1, seis responderam com mais de 50\% do Valor de Importância (VI) total (Tabela 3), destacando-se Byrsonima sericea com 9,2\% da densidade relativa e $18,5 \%$ da frequência relativa, seguida por Tapirira guianensis (14,2\% e 8,0\%), Eriotheca crenulaticalyx (1,6\% e $20,2 \%)$, Thyrsodium schomburgkianum (12\% e 1,2\%) e Artocarpus heterophyllus (8\% e 8,3\%). Tapirira guianensis ainda se destacou com a maior frequência relativa do N1. Eriotheca crenulaticalyx, espécie com o terceiro maior valor importância para o N1, possui a maior dominância relativa em função da área basal de oito indivíduos presentes nesta altitude (Tabela 3).

Tabela 3 - Espécies arbóreas amostradas no levantamento estrutural nos três níveis altitudinais no Brejo dos Cavalos, Pernambuco, Brasil com seus respectivos descritores fitossociológicos. Organizada de acordo com a ordem decrescente de VI

Table 3 - Tree species shown in the structural survey in the three altitudinal levels in the Brejo dos Cavalos, Pernambuco state, Brazil with their phytosociological parameters. Organized according to IV decreasing order

\begin{tabular}{|c|c|c|c|c|c|c|c|c|c|c|c|c|}
\hline \multirow[t]{2}{*}{ Espécies } & \multicolumn{4}{|c|}{ Nível 1} & \multicolumn{4}{|c|}{ Nível 2} & \multicolumn{4}{|c|}{ Nível 3} \\
\hline & DR & FR & DoR & VI & DR & FR & DoR & VI & DR & FR & DoR & VI \\
\hline Byrsonima sericea & 9,2 & 8,1 & 18,54 & 36,25 & 12,6 & 11,31 & 24,26 & 48,16 & 3,4 & 4,23 & 6,44 & 14,07 \\
\hline $\begin{array}{l}\text { Eriotheca } \\
\text { crenulaticalyx }\end{array}$ & 1,6 & 2,13 & 20,2 & 23,92 & 8,0 & 7,54 & 27,67 & 43,21 & 4,6 & 5,47 & 11,71 & 21,78 \\
\hline Tapirira guianensis & 14,2 & 12,77 & 8,01 & 34,98 & 20,2 & 16,08 & 5,93 & 42,21 & 20,2 & 16,17 & 6,69 & 43,06 \\
\hline Guapira laxiflora & 3,0 & 3,19 & 2,4 & 8,59 & 3,8 & 4,52 & 2,28 & 10,6 & 10,2 & 8,71 & 8,13 & 27,04 \\
\hline $\begin{array}{l}\text { Thyrsodium } \\
\text { schomburgkianum }\end{array}$ & 12,0 & 9,57 & 1,2 & 22,77 & 0,8 & 0,75 & 0,27 & 1,82 & 3,0 & 3,23 & 0,44 & 6,68 \\
\hline Ocotea glomerata & 6,4 & 5,32 & 1,96 & 13,68 & 7,4 & 8,79 & 3,7 & 19,9 & 9,0 & 9,45 & 4,1 & 22,55 \\
\hline Eugenia punicifolia & 3,6 & 4,79 & 1,07 & 9,46 & 0,4 & 0,5 & 0,44 & 1,34 & 7,0 & 6,47 & 5,36 & 18,83 \\
\hline Ormosia fastigiata & 2,6 & 2,39 & 2,04 & 7,03 & 5,6 & 5,78 & 6,88 & 18,26 & 1,6 & 1,74 & 1,17 & 4,51 \\
\hline Inga sp. & 6,4 & 7,18 & 2,98 & 16,56 & 0,2 & 0,25 & 0,01 & 0,46 & 0,6 & 0,75 & 0,43 & 1,77 \\
\hline $\begin{array}{l}\text { Stryphnodendron } \\
\text { pulcherrimum }\end{array}$ & 1,6 & 1,86 & 3,86 & 7,32 & 1,8 & 2,26 & 2,01 & 6,07 & 1,6 & 1,99 & 10,86 & 14,45 \\
\hline Casearia sylvestris & 6,2 & 6,12 & 1,01 & 13,33 & 6,0 & 6,78 & 1,38 & 14,17 & 2,4 & 2,49 & 0,55 & 5,43 \\
\hline Swartzia pickelli & 1,0 & 1,06 & 7,58 & 9,64 & 2,2 & 2,76 & 8,37 & 13,34 & 0,6 & 0,75 & 3,02 & 4,37 \\
\hline Cordia sellowiana & 0,4 & 0,53 & 0,68 & 1,62 & 0,4 & 0,5 & 0,02 & 0,92 & 2,4 & 2,49 & 8,23 & 13,12 \\
\hline Myrcia sylvicata & 1,8 & 2,39 & 0,5 & 4,69 & 5,8 & 6,03 & 0,64 & 12,48 & 1,8 & 1,74 & 0,22 & 3,76 \\
\hline Clusia dardanoi & 0,6 & 0,8 & 0,18 & 1,58 & 5,8 & 5,78 & 0,74 & 12,32 & 3,6 & 3,98 & 0,62 & 8,2 \\
\hline Nectandra cuspidata & 3,2 & 3,99 & 3,6 & 10,79 & 0,6 & 0,75 & 0,06 & 1,42 & 3,4 & 3,23 & 2,83 & 9,46 \\
\hline
\end{tabular}


Tabela 1 - Continuação...

Table 1 - Continuation...

\begin{tabular}{|c|c|c|c|c|c|c|c|c|c|c|c|c|}
\hline \multirow[t]{2}{*}{ Espécies } & \multicolumn{4}{|c|}{ Nível 1} & \multicolumn{4}{|c|}{ Nível 2} & \multicolumn{4}{|c|}{ Nível 3} \\
\hline & DR & FR & DoR & VI & DR & FR & DoR & VI & DR & FR & DoR & VI \\
\hline Albizia polycephala & 0,2 & 0,27 & 0,55 & 1,02 & 2,2 & 2,26 & 2,27 & 6,74 & 1,2 & 1,24 & 0,07 & 2,51 \\
\hline Guarea macrophylla & 2,6 & 2,93 & 0,93 & 6,46 & 0,4 & 0,5 & 0,02 & 0,83 & 1,4 & 1,49 & 0,15 & 3,04 \\
\hline Simarouba amara & 0,8 & 1,06 & 2,4 & 4,27 & 1,2 & 1,26 & 3,46 & 5,92 & 0,4 & 0,5 & 1,83 & 2,73 \\
\hline Machaerium hirtum & 0,4 & 0,53 & 0,03 & 0,96 & 1,4 & 1,26 & 1,49 & 4,14 & 0,6 & 0,75 & 0,26 & 1,61 \\
\hline $\begin{array}{l}\text { Erythroxylum } \\
\text { mucronatum }\end{array}$ & 0,2 & 0,27 & 0,01 & 0,48 & 1,2 & 1,26 & 0,21 & 2,66 & 0,4 & 0,5 & 0,07 & 0,97 \\
\hline Andira nitida & 0,4 & 0,53 & 0,08 & 1,01 & 0,2 & 0,25 & 0,18 & 0,63 & 0,4 & 0,5 & 1,51 & 2,41 \\
\hline Palicourea crocea & 0,4 & 0,53 & 0,03 & 0,96 & 0,2 & 0,25 & 0,01 & 0,46 & 0,6 & 0,75 & 0,09 & 1,44 \\
\hline Genipa americana & 0,2 & 0,27 & 0,17 & 0,64 & 0,6 & 0,75 & 0,04 & 1,39 & 0,4 & 0,5 & 0,27 & 1,17 \\
\hline Coffea arabica & 0,4 & 0,53 & 0,02 & 0,95 & 0,4 & 0,5 & 0,02 & 0,93 & 0,6 & 0,75 & 0,04 & 1,39 \\
\hline Artocarpus heterophyllus & 8,0 & 5,59 & 8,29 & 21,87 & 4,2 & 3,77 & 3,18 & 11,14 & & & & \\
\hline Cecropia pachystachya & 4,4 & 5,59 & 4,85 & 14,83 & 0,8 & 0,75 & 0,6 & 2,15 & & & & \\
\hline Combretum sp. & 0,2 & 0,27 & 0,02 & 0,49 & 1,2 & 1,51 & 0,88 & 3,59 & & & & \\
\hline Vochysia sp. & 0,8 & 0,8 & 0,25 & 1,85 & 0,2 & 0,25 & 0,01 & 0,46 & & & & \\
\hline Morfoespécie 1 & 0,8 & 0,8 & 0,24 & 1,83 & 0,2 & 0,25 & 0,08 & 0,53 & & & & \\
\hline Micropholis sp. & & & & & 1,0 & 1,26 & 0,68 & 2,93 & 4,2 & 3,98 & 7,9 & 16,08 \\
\hline Podorcarpus sellowii & & & & & 0,2 & 0,25 & 0,01 & 0,46 & 2 & 1,74 & 4,8 & 8,55 \\
\hline Miconia munutiflora & & & & & 1,4 & 1,51 & 0,26 & 3,16 & 3 & 3,48 & 1,65 & 8,13 \\
\hline Schefflera morototoni & & & & & 0,6 & 0,75 & 0,04 & 1,4 & 0,8 & 1,0 & 0,08 & 1,87 \\
\hline Morfoespécie 2 & 0,2 & 0,27 & 0,03 & 0,5 & & & & & 1,4 & 1,49 & 1,2 & 4,09 \\
\hline Zanthoxylum rhoifolium & 0,6 & 0,8 & 0,08 & 1,48 & & & & & 1,2 & 1,49 & 1,09 & 3,78 \\
\hline Vismia guianensis & 1,6 & 1,6 & 0,13 & 3,32 & & & & & 1,0 & 1,0 & 0,15 & 2,14 \\
\hline Cedrela fissilis & 0,8 & 1,06 & 0,28 & 2,15 & & & & & 0,4 & 0,5 & 1,96 & 2,86 \\
\hline Attalea oleífera & 0,2 & 0,27 & 0,98 & 1,45 & & & & & 0,4 & 0,5 & 0,02 & 0,92 \\
\hline Schinopsis brasiliensis & 0,2 & 0,27 & 0,25 & 0,72 & & & & & 0,4 & 0,5 & 0,08 & 0,97 \\
\hline $\begin{array}{l}\text { Anacardium } \\
\text { occidentale }\end{array}$ & 0,8 & 0,8 & 2,22 & 3,82 & & & & & & & & \\
\hline Cytharexylum sp. & 0,6 & 0,53 & 1,08 & 2,21 & & & & & & & & \\
\hline Byrsonima crispa & 0,2 & 0,27 & 0,65 & 1,12 & & & & & & & & \\
\hline Melochia sp. & 0,4 & 0,53 & 0,09 & 1,02 & & & & & & & & \\
\hline Piptadenia stipulacea & 0,2 & 0,27 & 0,45 & 0,92 & & & & & & & & \\
\hline Andira fraxinifolia & 0,2 & 0,27 & 0,01 & 0,48 & & & & & & & & \\
\hline Talisia esculenta & 0,2 & 0,27 & 0,01 & 0,48 & & & & & & & & \\
\hline
\end{tabular}


Tabela 3 - Conclusão...

Table 3 - Conclusion...

\begin{tabular}{|c|c|c|c|c|c|c|c|c|c|c|c|c|}
\hline \multirow[t]{2}{*}{ Espécies } & \multicolumn{4}{|c|}{ Nível 1} & \multicolumn{4}{|c|}{ Nível 2} & \multicolumn{4}{|c|}{ Nível 3} \\
\hline & DR & FR & DoR & VI & DR & FR & DoR & VI & DR & FR & DoR & VI \\
\hline Buchenavia capitata & & & & & 0,2 & 0,25 & 1,05 & 1,5 & & & & \\
\hline Aspidosperma sp. & & & & & 0,2 & 0,25 & 0,78 & 1,23 & & & & \\
\hline Ficus cf. maxima & & & & & & & & & 0,6 & 0,75 & 3 & 4,35 \\
\hline Ocotea gardneri & & & & & & & & & 1,2 & 1,49 & 0,76 & 3,45 \\
\hline Pterocarpus violace & & & & & & & & & 0,4 & 0,5 & 1,91 & 2,81 \\
\hline Mabea occidentalis & & & & & & & & & 1,2 & 1,24 & 0,26 & 2,7 \\
\hline Copaifera langsdorffii & & & & & & & & & 0,2 & 0,25 & 0,01 & 0,46 \\
\hline Licania octandra & & & & & & & & & 0,2 & 0,25 & 0,01 & 0,46 \\
\hline
\end{tabular}

Em que: $\mathrm{DR}$ = densidade relativa (\%); FR = frequência relativa (\%); DoR = dominância relativa (\%); IVI = índice de valor de importância.

No nível intermediário, mais de $50 \%$ do VI total pertenciam a apenas quatro espécies: Byrsonima sericea, Eriotheca crenulaticalyx, Tapirira guianensis e Ocotea glomerata. Em relação aos parâmetros relativos, Swartzia pickelli e Ormosia fastigiata, com 11 e 28 indivíduos, respectivamente, representaram o terceiro e quarto maior valor de dominância relativa (Tabela 3), o que indica a presença de alguns indivíduos de grande porte nesse nível. Por outro lado, foram amostrados 101 indivíduos de Tapirira guianenses neste nível, entretanto, com dominância relativa menor que as duas espécies anteriormente citadas, o que revela o pequeno porte de indivíduos de T. guianenses no N2. Tapirira guianenses apresenta-se com o terceiro maior VI devido à maior frequência e densidade relativa.

Das espécies amostradas no N3, seis responderam aproximadamente $50 \%$ do VI deste nível, com destaque novamente para $T$. guianensis com $20,2 \%$ de densidade relativa e $6,69 \%$ de dominância relativa, seguida por Guapira laxiflora com 10,2\% e 8,13\% para os mesmos descritores respectivamente. Tapirira guianensis também apresenta a maior frequência relativa no N3. Eugenia punicifolia apresentou 35 indivíduos neste nível, valor diferente ao N2, sendo amostrados apenas dois indivíduos. Destaca-se ainda, a alta dominância relativa de Stryphnodendron pulcherrimum em relação ao pequeno número de representantes (oito indivíduos) no N3 (Tabela 3).

Em relação aos parâmetros estruturais, houve uma grande variação nos valores de densidade entre os níveis altitudinais N1, N2 e N3 (780; 3069 e 278 indivíduos/ha, respectivamente). A variação dos valores de densidade corrobora outros estudos como o de Meireles, Shepherd e Kinoshita (2008) em que os autores também encontraram os menores valores de densidade nos extremos do gradiente, entretanto, contrapõe os estudos de Gomes, Bernacci e Joly (2011) que observaram uma relação direta entre densidade e altitude. A maior densidade no N2 pode ser atribuída à maior concentração de indivíduos de menores valores médios de altura e diâmetro (10,2 metros e 42,61 cm), devido ao histórico precedente de extração de terra, madeira e cultivo de hortaliças nessa porção intermediária, segundo moradores locais. Por outro lado, a área basal não variou entre os níveis de elevação $\left(15,71 ; 15,06\right.$ e 14,92 $\mathrm{m}^{2} /$ ha, respectivamente). Os valores de área basal encontrados são muito baixos quando comparadas a outras áreas de floresta estacional semidecidual, como por exemplo, um estudo realizado em um Parque Municipal em Minas Gerais apresentou uma área basal total de $23,18 \mathrm{~m}^{2} /$ ha, atribuindo este valor ao processo de perturbação que ainda ocorre dentro do parque (CORAIOLA; NETTO, 2017). Estudos realizados em ecossistemas serranos, demonstram cada vez mais que estas florestas não apresentam um 
padrão recorrente na organização florística e estrutural, Espiríto-Santo et al. (2002) sugerem que as variáveis associadas à organização espacial das comunidades vegetais em áreas serranas mudam caso a caso.

Tapirira guianensis foi a segunda espécie de maior VI no N1, no entanto, apresenta-se no N2 como quarta espécie de maior VI, em função da elevada dominância relativa de Byrsonima sericea e Eriotheca crenulaticalyx para esta altitude. Já no N3, Tapirira guianensis apresenta-se com o maior VI e as duas espécies anteriormente citadas nesta altitude, apresentam o oitavo e quarto lugar, respectivamente (Figura 3).

\section{Figura 3 - Posicionamento das espécies comuns a todas as altitudes em relação ao VI nos diferentes níveis altitudinais do Brejo dos Cavalos, Pernambuco, Brasil}

Figure 3 - Positioning species common to all altitudes relative to VI the different altitudinal levels of the Brejo dos Cavalos, Pernambuco state, Brazil

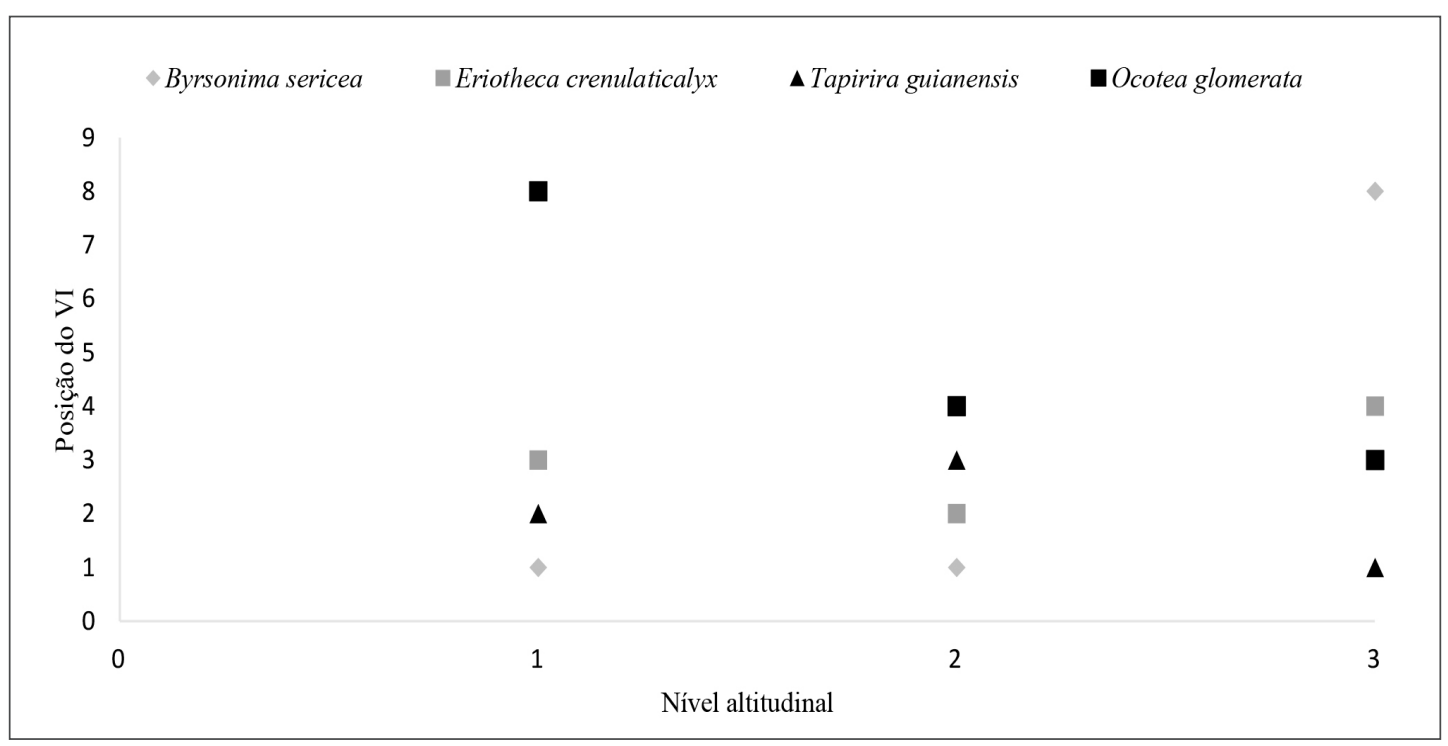

Fonte: Thallyta Guimarães de Araujo, et al. 2018

Com relação à Diversidade de Shannon-Wiener ( $\left.H^{\prime}\right)$ não houve diferenças significativas entre N1 e N3 (3,02 e 3,06; $P>0,05)$, o que pode ser constatado pela diferença discreta nos valores de riqueza de espécies entre esses níveis de altitudes (44 e 41 espécies, respectivamente). Porém, o nível intermediário $\left(\mathrm{H}^{\prime}=2,84\right)$ possui uma variação significativa quando comparado ao $\mathrm{N} 1 \mathrm{e}$ N3 $(P<0,05)$, devido à baixa riqueza de espécies e alta abundância nesse nível, principalmente a dominância de Tapirira guianensis e Byrsonima sericea. Por mais pequeno que seja, um fragmento pode conter várias peças de mosaico de vegetação relacionadas a diferenças de substrato e/ou a fase sucessional (ESPIRITO-SANTO et al., 2002), nesse sentido, o fragmento aqui analisado apresenta abertura de clareiras, trilhas e corpos d'água (açude), principalmente na porção intermediária, nos quais foi identificada a presença de espécies exóticas como a jaca (Artocarpus heterophyllus) e o café (Coffea arabica), o que pode resultar em diferentes estágios de sucessão ao longo do gradiente. Levando-se em consideração estas informações, as maiores riquezas no N1 e N3 podem estar relacionadas tanto ao maior número de espécies exclusivas em cada um desses níveis de altitude, sendo oito e seis espécies, respectivamente (Figura 2), quanto aos diferentes estágios de conservação. A baixa densidade do N3, por exemplo, possivelmente deve-se aos maiores portes dos indivíduos nesse nível, em que florestas mais maduras possuem fisionomia mais preservadas, com árvores de maior altura e diâmetro o que reflete em um estágio sucessional mais avançado (SILVA et al., 2015). Estas características do N3 também podem ser observadas 
pelo maior valor de equabilidade nesta faixa de altitude, o que indica uma distribuição mais uniforme dos indivíduos nas espécies, aumentado o valor de diversidade. Já o N2 apresenta um menor número de espécies exclusivas, o que reflete em uma menor riqueza, aliando a isso, a presença de perturbações e intervenções antrópicas neste nível, pode estar levando a uma dominância de Byrsonima sericea, Eriotheca crenulaticalyx e Tapirira guianensis, dificultando, assim, o estabelecimento de outras espécies devido à competição por recursos (HOMEIER et al., 2010; KRAFT et al., 2011).

Das dez espécies de maior VI para cada nível, quatro são comuns às três altitudes (Byrsonima sericea, Eriotheca crenulaticalyx, Tapirira guianensis e Ocotea glomerata). Estas espécies também foram amostradas com elevada importância em outros brejos de altitudes (ANDRADE et al., 2006; OLIVEIRA; ANDRADE; FELIX, 2006; NASCIMENTO; RODAL, 2008; PINTO; SAMPAIO; NASCIMENTO 2012). Byrsonima sericea apresenta-se no N1 e N2 como a espécie mais importante e ocupa a oitava posição no N3, principalmente, em função da baixa dominância relativa, que pode ser explicado pela redução de sua área basal na maior altitude. Essa espécie apresenta se na base da serra (N1) com uma área basal de $2,96 \mathrm{~m}^{2}$, no nível intermediário (N2) com $3,98 \mathrm{~m}^{2}$ e na porção mais alta da serra (N3) com apenas $0,91 \mathrm{~m}^{2}$. Mudanças nos valores de área basal podem estar relacionado diretamente à variação na abundância (CORAIOLA; NETTO, 2017), como é o caso registrado no presente estudo. Eriotheca crenulaticalyx possui os maiores valores de área basal para as três altitudes.

Tapirira guianensis é a espécie mais abundante na serra como um todo e também em cada uma das altitudes investigadas, o que a faz ser a espécie com maior sucesso em explorar os recursos ao longo de todo o gradiente altitudinal. Esta espécie se enquadra no padrão de espécies cuja densidade varia localmente em função da extensão de seus habitat preferenciais (DURIGAN et al., 2000). No entanto, é considerada generalista por habitat (OLIVEIRA FILHO; RATTER, 1995; 2000) e dependente de vetores bióticos para sua reprodução sexuada, por outro lado, tais polinizadores são eficientes mesmo em áreas antropizadas (BOEGER et al., 1998). Desta forma, a eficiência da reprodução sexuada de Tapirira guianenses pode explicar a ampla distribuição e importância ecológica da espécie (BOEGER et al., 1998) na serra.

Essa alternância de dominância das espécies, evidencia a existência de diferentes microhabitats entre os níveis de altitude. Urbanetz e colaboradores (2013), em um gradiente de altitude, observaram diferenças na estrutura e na importância das espécies ao longo do gradiente, coincidindo com uma mudança no tipo de formação vegetal. No entanto, o Brejo dos Cavalos apresenta uma única formação vegetal ao longo de toda a serra, o que pode ser evidenciado pelo fato dessas quatro espécies (Byrsonima sericea, Eriotheca crenulaticalyx, Tapirira guianensis e Ocotea glomerata) serem apontadas como as mais importantes na serra como todo, porém, apresentando uma alternância, entre elas, em relação ao VI nos diferentes níveis de altitude, o que pode ter sido propiciado por diferenças topográficas e fatores antrópicos.

\section{Conclusão}

O Brejo de altitude Serra dos Cavalos exibiu uma unidade florística comum, no entanto, apresentou diferenças nos parâmetros estruturais das comunidades entre os níveis de altitude. Apesar de não terem sido realizadas análises de fatores abióticos e de impactos antrópicos, tais diferenças podem ser atribuídas às variações das características ambientais a cada nível de altitude, principalmente relacionadas à maior frequência de perturbações no nível intermediário, o que proporcionou menor diversidade e maior densidade neste nível. Nesse sentido, a variação dos descritores fitossociológicos entre os níveis também é consequência da dominância ecológica diferenciada (maiores VI) de Byrsonima sericea, Eriotheca crenulaticalyx, Tapirira guianensis e Ocotea glomerata ao longo do gradiente. Essas espécies seriam as que realmente definiram a estrutura dessa floresta de brejo, já que tais espécies apresentaram uma vantagem seletiva sobre as outras e passaram a ocupar um lugar representativo no ambiente, o que pode ser confirmado 
pelos descritores fitossociológicos, com mudanças na posição do VI de acordo com o nível de altitude.

\section{Referências}

AGÊNCIA ESTADUAL DE MEIO AMBIENTE E RECURSOS HÍDRICOS (PE). Diagnóstico para recuperação do Parque Ecológico João Vasconcelos-Sobrinho. Recife, 1994. (Série Biblioteca do Meio Ambiente, 1).

ANGIOSPERM PHYLOGENY GROUP III. An update of the Angiosperm Phylogeny Group classification for the orders and families of flowering plants: APG III. Botanical Journal of the Linnean Society, London, v. 161, n. 2, p. 105-121, 2009.

ANDRADE, L. A. et al. Análise florística e estrutural de matas ciliares de ocorrentes em brejo de altitude, no município de Areia, Paraíba. Revista Brasileira de Ciências Agrárias, Recife, v. 1, p. 31-40, 2006.

BOEGER, M. R. et al. Variações morfo-anatômicas dos folíolos de Tapirira guianensis Aubl. em relação a diferentes estratos da floresta. Biotemas, Florianópolis, v. 11, n. 2, p. 27-38, 1998.

BOTREL, R. T. et al. Influência do solo e topografia sobre as variações da composição florística e estrutura da comunidade arbóreo-arbustiva de uma floresta estacional semidecidual em Ingaí, MG. Revista Brasileira de Botânica, São Paulo, v. 25, n. 2, p. 195-213, 2002.

BRAGA, A. J. T.; LIMA, E. E.; MARTINS, S. V. Influência dos fatores edáficos na variação florística de Floresta Estacional Semidecidual, em Viçosa, MG. Revista Árvore, Viçosa, MG, v. 39, n. 4, p.623-633, 2015.

CARVALHO, D. A. et al. Variações florísticas e estruturais do componente arbóreo de uma Floresta Ombrófila Alto-Montana às margens do rio Grande, Bocaina de Minas, MG, Brasil. Acta Botânica Brasilica, Belo Horizonte, v. 19, n. 1, p. 91-109, 2005.

CAVALCANTI, D. R.; TABARELLI, M. Distribuição das plantas amazônico-nordestinas no centro de endemismo Pernambuco: brejos de altitude vs. florestas de terras baixas. In: PÔRTO, K. C.; CABRAL, J. J. P.; TABARELLI, M. (Ed.). Brejos de altitude em Pernambuco e Paraíba: história natural, ecologia e conservação. Brasília: Ministério do Meio Ambiente, 2004. p. 285-296.

CESTARO, L. A.; SOARES, J. J. Variações florística e estrutural e relações fitogeográficas de um fragmento de floresta decídua no Rio Grande do Norte, Brasil. Acta Botanica Brasilica, Belo Horizonte, v. 18, n. 2, p. 203-218, 2004.

CORAIOLA, M.; NETTO, S. P. Análise da estrutura dimensional de uma floresta estacional semidecidual localizada no município de Cássia-MG: estrutura volumétrica. Revista Acadêmica: Ciência Animal, Curitiba, v. 1, n. 4, p. 11-24, 2017.

COTTAM, G.; CURTIS, J. T. The use of distance measures in phytosociological sampling. Ecology, Washington, v. 37, n. 45, p. 1-460, 1956.

DUQUE, A. et al. Distance Decay of tree species similarity in protected areas on terra firme forests in Colombian Amazonia. Biotropica, Gainesville, v. 10, p. 1-9, 2009.

DURIGAN, G. et al. Estrutura e diversidade do componente arbóreo da floresta na Estação Ecológica dos Caetetus, Gália, SP. Revista Brasileira de Botânica, São Paulo, v. 23, n. 4, p. 371383,2000 .

ESPÍRITO-SANTO, F. D. B. et al. Variáveis ambientais e a distribuição de espécies arbóreas em um remanescente de floresta estacional semidecídua montana no campus da Universidade Federal de Lavras, MG. Acta Botanica Brasilica, Belo Horizonte, v. 16, n. 3, p. 331-356, 2002.

FARIAS, S. G. G. et al. Physiognomy and vegetation structure in different environments of "caatinga" in" Serra Talhada" Pernambuco State, Brazil/Fisionomia e estrutura de vegetação de caatinga em diferentes ambientes em Serra Talhada--Pernambuco. Ciência Florestal, Santa Maria, v. 26, n. 2, p. 435-449, 2016. 
FUNDAÇÃO SOS MATA ATLÂNTICA. Mapa de remanescentes da Floresta Atlântica nordestina. In: SOCIEDADE NORDESTINA DE ECOLOGIA, CONSERVATION INTERNATIONAL; FUNDAÇÃO BIODIVERSITAS (Ed.). Workshop Prioridades para a Conservação da Floresta Atlântica do Nordeste. Recife, 1993.

GENTRY, A. H. Changes in plant community diversity and floristic composition on environmental and geographical gradients. Annals of Missouri Botanical Garden, Missouri, v. 75, p. 1-34, 1988.

GOMES, J. A. M. A., BERNACCI, L. C.; JOLY, C. A. Floristic and structural differences, between two altitudinal quotas, of the Submontane Atlantic Rainforest within the Serra do Mar State Park, municipality of Ubatuba/SP, Brazil. Biota Neotropica, Campinas, v. 1, n. 2, p. 123-137, 2011.

HAMMER, O.; HARPER, D. T. A.; RYAN, P. D. PAST: Paleontological Statistics software package for education and data analysis. Paleontologia Electronica, California, v. 4, n. 1, p. 9, 2001.

HOMEIER, J. et al. Tree Diversity, Forest Structure and Productivity along Altitudinal and Topographical Gradients in a Species-Rich Ecuadorian Montane Rain Forest. Biotropica, Gainesville, v. 42, n. 2, p. 140-148, 2010.

IBGE. Manual Técnico da Vegetação Brasileira. 2. ed. Rio de Janeiro, 2012. (Manuais Técnicos em Geociências, 1).

IVANAUSKAS, N. M.; MONTEIRO, R.; RODRIGUES, R. R. Similaridade florística entre áreas de Floresta Atlântica no Estado de São Paulo. Brazilian Journal of Ecology, São Paulo, v. 1, n. 1, p. 71-81, 2000.

KELLMAN, M. Geographic patterning in tropical weed communities and early secondary successions. Biotropica, Gainesville, p. 34-39, 1980.

KRAFT, N. J. B. et al. Disentangling the drivers of $\square$ diversity along latitudinal and elevational gradients. Science, Washington, v. 333, n. 6050, p. 1755-1758, 2011.

LOPES, S. F. et al. Historical review of studies in seasonal semideciduous forests in Brazil: a perspective for conservation. Brazilian Geographical Journal: Geosciences and Humanities Research Medium, Uberlândia, v. 2, p. 21-40, 2012.

LOPES, S. F.; RAMOS, M. B.; ALMEIDA G. R. The Role of Mountains as Refugia for Biodiversity in Brazilian Caatinga: Conservationist Implications. Tropical Conservation Science, San Andrés, v. 10, p. 1-12, 2017.

MEIRELES, L. D.; SHEPHERD, G. J.; KINOSHITA, L. S. Variações na composição e na estrutura fitossociológica de uma floresta ombrófila densa alto-montana na Serra da Mantiqueira, Monte Verde, MG. Revista Brasileira de Botânica, São Paulo, v. 31, n. 4, p. 559-574, 2008.

MONTEIRO, E. A.; FISCH, S. T. V. Estrutura e padrão espacial das populações de Bactris setosa Mart. e B. hatschbachii Noblick ex A. Hend (Arecaceae) em um gradiente altitudinal, Ubatuba (SP). Biota Neotropica, Campinas, v. 5, n. 2, p. 1-7, 2005.

MORELLATO, L. P. C. The Brazilian Atlantic Forest. Biotropica, Gainesville, v. 32, n. 4b, p. 786$792,2000$.

NASCIMENTO, L. M.; RODAL, M. J. N. Fisionomia e estrutura de uma floresta estacional montana do maciço da Borborema, Pernambuco - Brasil. Revista Brasileira de Botânica, São Paulo, v. 31, n. 1, p. 27-39, 2008.

OLIVEIRA, A. A.; MORI, S. A. A. central Amazonian terra firme Forest. I: high tree species richness on poor soils. Biodiversity and Conservation, Holanda, v. 8, n. 9, p. 1219-1244, 1999.

OLIVEIRA, F. X.; ANDRADE, L. A.; FELIX, L. P. Comparações florísticas e estruturais entre comunidades de floresta ombrófila aberta com diferentes idades, no município de Areia, PB, Brasil. Acta Botânica Brasilica, Belo Horizonte, v. 20, n. 4, p. 861-873, 2006.

OLIVEIRA, P. T. B. et al. Florística e fitossociologia de quatro remanescentes vegetacionais em áreas de serra no cariri paraibano. Revista Caatinga, Mossoró, v. 22, p. 169-178, 2009. 
OLIVEIRA FILHO, A. T. et al. Estrutura fitossociológica e variáveis ambientais em um trecho da mata ciliar do córrego dos Vilas Boas, Reserva Biológica do Poço Bonito, Lavras (MG). Revista Brasileira de Botânica, São Paulo, v. 17, p. 67-85, 1999.

OLIVEIRA FILHO, A. T.; RATTER, J. A. A study of the origin of central Brazilian forests by the analysis of plant species distribution patterns. Edinburgh Journal of Botany, Cambridge, v. 52, n. 2, p. 141-194, 1995.

OLIVEIRA FILHO, A. T.; RATTER, J. A. Padrões florísticos das matas ciliares da região do cerrado e a evolução das paisagens do Brasil Central durante o Quaternário Tardio. In: MATAS ciliares: conservação e recuperação. São Paulo: EDUSP, 2000. v. 2. p. 73-89.

PEEL, M. C.; FINLAYSON, B. L.; MCMAHON, T. A. Updated world map of the Köppen-Geiger climate classification. Hidrology and Earth System Sciences Discussions, Munique, v. 4, p. 439-473, 2007.

PINTO, M. S. C.; SAMPAIO, E. V. S. B.; NASCIMENTO, L. M. Florística e estrutura da vegetação de um brejo de altitude em Pesqueira, PE, Brasil. Revista Nordetina de Biologia, João Pessoa, v. 21, n. 1, p. 47-79, 2012.

PROCTOR, J. et al. Ecological studies on Gunung Silam, a small ultrabasic mountain in Sabah, Malaysia. Journal of Ecology, London, v. 76, n. 2, p. 320-340, 1988.

SHEPHERD, G. J. Fitopac. File Version 2.1.2.85. Campinas: Unicamp, 2010.

SILVA, W. G. et al. Relief influence on tree species richness in secondary forest fragments of Atlantic Forest, SE, Brazil. Acta Botanica Brasilica, Belo Horizonte, v. 22 n. 2. p. 289-298, 2008.

SILVA, F. K. G. et al. Patterns of species richness and conservation in the Caatinga along elevational gradients in a semiarid ecosystem. Journal of Arid Environments, New York, v. 110, p. 47-52, 2014.

SILVA, M. A. M. et al. Does the plant economics spectrum change with secondary succession in the forest? Trees, Germany, v. 29, n. 5, p. 1521-1531, 2015.

TAVARES, M. C. G. et al. Fitossociologia do componente arbóreo de um trecho de floresta ombrófila montana do Parque Ecológico João Vasconcelos Sobrinho, Caruaru, Pernambuco. Naturalia, São Paulo, v. 25, p. 243-270, 2000.

TUOMISTO, H.; RUOLOLAINEN, K.; YLI-HALLA, M. Dispersal, environment, and floristic variation of western Amazonian forests. Science, Washington, v. 299, p. 241-244, 2003.

URBANETZ, C. et al. Composição e distribuição de espécies arbóreas em gradiente altitudinal, Morraria do Urucum, BRASIL. Oecologia Australis, Rio de Janeiro, v. 16, n. 4, p. 859-877, 2013.

VASCONCELOS SOBRINHO, J. Os brejos de altitude e as matas serranas. In: PERNAMBUCO. As regiões naturais do Nordeste, o meio e a civilização. Recife: Conselho de Desenvolvimento de Pernambuco, 1970. p. 79-86.

WEBB, C. O.; PEART, D. R. Habitat associations of trees and seedlings in a Bornean rain forest. Journal of Ecology, London, v. 88, n. 3, p. 464-478, 2000.

WHITTAKER, R. J.; WILLIS, K. J.; FIELD, R. Scale and species richness: towards a general, hierarchical theory of species diversity. Journal of Biogeography, Malden, v. 28, n. 4, p. 453-470, 2001.

ZHAO, C. M. et al. Altitudinal pattern of plant species diversity in Shennongjia Mountains, Central China. Journal of Integrative Plant Biology, Malden, v. 47, p. 1431-1449, 2005. 